\title{
Otimização de Rede Coletora Interna de Parques Eólicos Offshore - Perspectivas e Funções de Mérito
}

\author{
Frederick T. Marliere*1. Vinícius A. Cabral*2. Frederico F. Panoeiro *3. Gustavo da S. Rebelo*4. \\ Leonardo W. Oliveira*5. Ivo C. S. Junior*6 \\ Departamento de Energia Elétrica, Universidade Federal de Juiz de Fora, Juiz de Fora, Brazil \\ *1frederick.tavares@engenharia.ufjf.br *2albuquerque.vinicius@engenharia.ufjf.br \\ *3frederico.panoeiro@engenharia.ufjf.br*4gustavo.rebello@engenharia.ufjf.br \\ *sleonardo.willer@ufjf.edu.br*6ivo.junior@ufjf.edu.br
}

\begin{abstract}
The present work presents perspectives and merit functions for the optimization of the internal electrical network topology of an offshore wind farm by using a bat echolocation algorithm. The optimization goals are the minimization of cable costs and the maximization of reliability, through the determination of radial topologies that meet such criteria. In order to evaluate reliability, a generation index specific for wind farms is used. The purpose is to evaluate solutions for the problem under different criteria through a comparative analysis by using data of test wind farm from the literature.
\end{abstract}

Resumo: O presente trabalho apresenta perspectivas e funções de mérito para a otimização da topologia de rede elétrica interna de um parque eólico offshore, através da utilização do algoritmo de ecolocalização de morcegos. Os objetivos considerados para otimização são a minimização dos custos de cabos e a maximização de confiabilidade, através da determinação de topologias radiais que atendam a estes critérios. Para avaliar confiabilidade, um índice de geração específico para parques eólicos é utilizado. O propósito é avaliar soluções para o problema sob diferentes critérios através de uma análise comparativa, utilizando-se dados de um parque eólico teste da literatura.

Keywords: Optimization; Wind Farm; Routing, Collection Network, Echolocation Algorithm.

Palavras-chaves: Otimização; Parque Eólico; Roteamento, Rede Coletora, Algoritmo de Ecolocalização.

\section{INTRODUÇÃO}

A energia cinética dos ventos como forma de geração de energia elétrica tem sido, a nível mundial, uma importante fonte energética renovável e limpa em crescente expansão. Em (Xiaoquing et al., 2011) considera-se a energia eólica como uma importante alternativa para a fuga da dependência dos combustíveis fósseis, e, com isso, constantes pesquisas nessa área levaram a um rápido e grande crescimento da penetração de energia eólica nos sistemas de energia mundiais. Segundo (WWEA, 2019), a capacidade de energia eólica em todo o mundo atinge $600 \mathrm{GW}$, sendo 53,9 GW adicionados somente no ano de 2018. No ano anterior, o acréscimo foi de 52,6 GW. Os dois países que lideram mundialmente em capacidade eólica são a China (200 GW) e os EUA (100 GW). Ainda segundo (WWEA, 2019), pode-se afirmar que, contando com toda a geração eólica presente mundialmente até o final de 2018, é possível cobrir cerca de $6 \%$ do total da demanda global de energia elétrica.

No Brasil, o cenário eólico é favorável e crescente. De acordo com (ABEEOLICA, 2019), a geração de energia eólica nacional cresceu $15 \%$ em 2018, tendo representatividade em $8,4 \%$ de toda energia produzida pelo Sistema Interligado Nacional, atingindo $15 \mathrm{GW}$ de capacidade instalada. O Rio Grande do Norte é o estado maior produtor de energia eólica produzindo 1.505,4 MW médios no ano de 2018. Ainda segundo (ABEEOLICA, 2019), a matriz eólica já representa a segunda fonte da matriz elétrica brasileira em capacidade instalada. Levantamentos de abril de 2019 indicam que o país contém mais de 7 mil aerogeradores, ou, do inglês, Wind Turbine (WT), em 601 parques eólicos. Esse cenário leva o país a ser $5^{\circ}$ colocado no ranking mundial de capacidade eólica nova onshore instalada em 2018 segundo dados globais divulgados pelo GWEC (Global Wind Energy Council).

Em (Mahidhar et al., 2008) e (Huang et al., 2009), afirma-se que o investimento total de um parque eólico offshore é tipicamente de $30 \%$ a $60 \%$ mais elevado do que um parque onshore com a mesma capacidade, entretanto existem vantagens como disponibilidade de ampla região de instalação bem como maior aproveitamento dos ventos incidentes. Diante desse aspecto pode-se perceber que ao realizar um investimento desse porte é necessário otimizar os aspectos relevantes de forma a diminuir os custos de operação e construção e maximizar o poder de geração eólica disponível.

Alguns trabalhos podem ser encontrados com o objetivo de otimizar as estruturas de parque eólico. Em (Li et al., 2008), buscou-se otimizar a topologia do sistema coletor de parques offshore, visando a minimização de custos com cabeamentos 
e manutenções, através do Algoritmo Genético associado com o Sistema Imunológico Artificial. Do ponto de vista da confiabilidade, essa otimização é primordial dado que o tipo de cabeamento interno da rede coletora define de forma direta seu impacto em confiabilidade. Segundo (Smail et al.,2018), há várias configurações possíveis que trazem complexidade proporcional ao tamanho do parque, o que amplia o espaço de busca de soluções e justifica o uso de técnicas inteligentes. Em (Wu et al., 2014), por sua vez, é proposta uma associação entre Algoritmo Genético e Ant Colony para otimizar a topologia da rede interna e também o posicionamento dos WT's, ou seja, o layout do parque eólico. A otimização de layout dos WT's também pode ser vista em (Yang et al., 2016) em um algoritmo de enxame de partículas que visa maximizar a potência extraída do parque eólico através da disposição dos mesmos.

O objetivo do presente trabalho é avaliar aspectos sobre a otimização da rede coletora de um parque eólico offshore sob diferentes perspectivas e funções de mérito relevantes para o problema. Assim, a partir de um layout previamente otimizado e estabelecido para o posicionamento dos WT's, é proposta a otimização do cabeamento interno da rede coletora, levando-se em consideração a minimização de custos com cabeamento e a maximização de confiabilidade. O propósito é avaliar o impacto destes diferentes critérios na decisão final sobre a estrutura de cabeamento interno do parque eólico. A otimização é realizada utilizando-se o algoritmo de ecolocalização de morcegos para resolução de problemas de programação inteira. Dados de um parque eólico teste da literatura são utilizados para a análise proposta.

\section{FORMULAÇÃO DO PROBLEMA}

Para a formulação do problema de otimização do roteamento interno de cabos em um parque eólico offshore, considera-se o layout de parque apresentado na Fig. 1, utilizado nas análises propostas no presente trabalho. Os dados deste parque podem ser encontrados em (Pookpunt et al., 2013), cuja a área total é de $4 \times 10^{6} \mathrm{~m}^{2}$, subdividida em 100 células, onde cada célula pode abrigar um WT. Partindo-se desta premissa, o layout da Fig. 1 é determinado em (Pookpunt et al., 2013), contando com $30 \mathrm{WT}$ 's, representados pelos pontos na figura, posicionados de forma a captar ventos na direção norte - sul. A subestação é representada pelo símbolo 'x', encontrando-se na posição $(\mathrm{x}=100 \mathrm{~m} \mathrm{e} \mathrm{y}=2200 \mathrm{~m})$. Os parâmetros técnicos considerados são dispostos na Tabela 1 .

Tabela 1. Parâmetros Considerados

\begin{tabular}{|c|c|}
\hline Diâmetro do rotor [m] & 40 \\
\hline Potência nominal do WT [kW] & 630 \\
\hline Altura em relação ao solo [m] & 60 \\
\hline Índice relativo à rugosidade do solo & 0,3 \\
\hline Coeficiente de impulso & 0,88 \\
\hline Velocidade do vento [m/s] & 12 \\
\hline Número de WT's & 30 \\
\hline
\end{tabular}

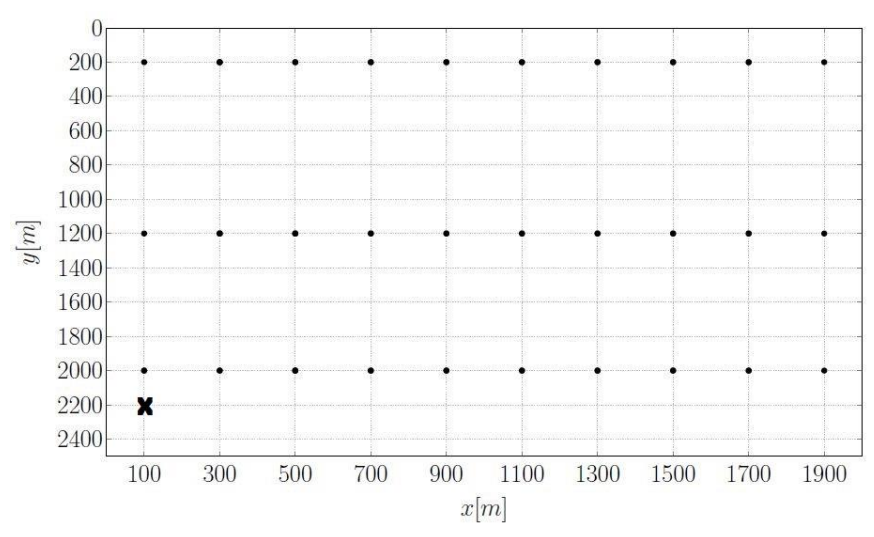

Fig. 1: Layout do parque eólico considerado.

A configuração da Fig. 1 foi obtida visando minimizar o custo de investimento nos WT's por potência extraída do parque eólico, considerando o efeito de esteira, ou do inglês, wake effect. São apresentadas a seguir as formulações para as funções de mérito consideradas no presente trabalho para o roteamento interno de parques eólicos.

\subsection{Minimização do Custo da Rede Coletora}

A formulação segundo o critério de custo da rede coletora visa modelar o problema de otimização do sistema coletor para uma topologia de WT's (localização) previamente determinada, como na Fig. 1 (Pookpunt et al., 2013). Em outras palavras, o propósito é conectar todos os pontos em que se localizam os WT's, direta ou indiretamente à subestação. O objetivo do critério de custos está associado à minimização do comprimento total dos cabos necessários para esta conexão, que implica no mínimo custo de roteamento, uma vez que todos os cabos são do mesmo tipo e capacidade de condução.

$\mathrm{O}$ cálculo do comprimento do cabo $\mathrm{L}_{12}$ entre WT's de coordenadas $\left(\mathrm{x}_{1}, \mathrm{y}_{1}\right)$ e $\left(\mathrm{x}_{2}, \mathrm{y}_{2}\right)$ é realizado utilizando-se a distância euclidiana entre os mesmos, conforme (1).

$$
L_{12}=\sqrt{\left(x_{1}-x_{2}\right)^{2}+\left(y_{1}-y_{2}\right)^{2}}
$$

O comprimento total $(L)$ dos cabos envolvidos no roteamento do parque eólico é dado pelo somatório dos comprimentos de cada cabo, conforme (2), em que $N$ é o número de conexões entre WT's.

$$
L=\sum_{i=1}^{N} L_{i}
$$

Dessa forma, pode-se formular a função objetivo $(F O B)$ para o critério de custos em função do comprimento total dos cabos da rede interna do parque eólico, conforme (3), em que Pen consiste numa penalidade aplicada, de valor 1000, escolhido empiricamente, quando alguma das restrições do problema é violada. Estas restrições são:

- A subestação deve estar conectada à rede elétrica interna do parque; 
- Não deve haver WT’s desconectados (ilhamento);

- $\quad$ Os WT's não devem ser conectados a si próprios.

Caso nenhuma das restrições anteriores seja violada, Pen recebe o valor unitário, significando ausência de penalidade.

$$
F O B=\operatorname{Min}(P e n \times L)
$$

\subsection{Maximização da Confiabilidade de Geração}

Segundo este critério, o objetivo é realizar a otimização da rede coletora maximizando a confiabilidade de geração. Para tanto, utiliza-se um índice de confiabilidade de rede, apresentado em (Menghua et al., 2007) próprio para parques eólicos offshore, denominado índice de taxa de disponibilidade de geração, ou do inglês Generation Ratio Avaibility (GRA). Esse índice mede a probabilidade de um percentual de energia eólica ser transferido para rede elétrica, uma vez que devido a falhas de elementos, a potência captada pelos WT's pode não ser totalmente transferida para a rede elétrica. Algumas vantagens deste índice são: $i$ ) não depende da carga ou demanda a ser atendida; ii) tem menor correlação com a velocidade do vento se comparado com outros índices de confiabilidade, sendo mais afetado pelas taxas de falha dos componentes de rede, como os cabos de conexão.

Outro índice, denominado taxa de geração, ou do inglês $G e$ neration Ratio $(G R)$, é utilizado para obter o $G R A$, sendo definido como a razão entre a potência (Psai) ou a energia (Esai) entregue à rede onshore e a potência (Pent) ou energia (Eent), respectivamente, total que o parque eólico pode fornecer. A Equação (4) formula o índice $G R$ considerando-se as potências entregues à rede onshore e máxima captada pelo parque.

$$
\text { GR = Psai } / \text { Pent }
$$

Tendo o índice $G R$ calculado, estabelece-se um valor crítico (mínimo aceitável) para que todos os estados analisados sejam divididos em dois grupos: $\{\mathrm{A}\}$ é o conjunto dos estados nos quais o valor de GR é maior ou igual ao GR crítico $\left(G R_{c}\right) ;\{\mathrm{B}\}$ é o conjunto dos estados analisados para os quais o valor obtido de GR é inferior ao valor de $G R_{c}$. Tendo ambos os conjuntos definidos, a relação da Equação (5) é estabelecida em (Menghua et al., 2007).

$$
G R A=P(G R \geq G R c)=\sum_{J=1}^{E}\left(\rho_{J} . \operatorname{Prob}\left\{A / w_{j}\right\}\right)
$$

Dessa forma, GRA representa a probabilidade do conjunto $\{\mathrm{A}\}$, em que $\rho_{j}$ é a probabilidade de ocorrência do estado de vento $j, E$ é o número total de estados de vento analisados e 'Prob $\left\{A / w_{j}\right\}$ ' é a probabilidade do conjunto $\{\mathrm{A}\}$ dado que o estado de vento $j$ está em análise.

A partir do roteamento ótimo obtido, avalia-se a confiabilidade do sistema coletor. Considera-se apenas um estado de vento com velocidade de $12 \mathrm{~m} / \mathrm{s}$ e direção nortesul. Assim, cada WT consegue prover uma determinada quantidade de potência, dadas as restrições de wake effect em função do layout (posicionamento dos WT's) previamente determinado. Para cada um dos $N$ cabos da rede elétrica, simula-se uma falha simples, que juntamente com o perfil de vento considerado, resulta em um estado para a análise de confiabilidade. Para cada estado, faz-se a análise do efeito de falha, do inglês Failure Effects Analysis (FEA), para verificar, mediante à falha em questão, quais WT's continuam conectados (ativos) e quais se desconectam da rede devido à falha em questão, tornando-se, portanto, inativos.

A partir da análise anterior, pode-se calcular o índice $G R$ para cada estado, uma vez que se sabe quais componentes estão ativos e quais estão inativos. Os estados analisados são separados nos grupos $\{\mathrm{A}\}$ e $\{\mathrm{B}\}$, considerando-se, no presente trabalho, $G R_{c}=0,8$. Desta forma, é possível calcular $\operatorname{Prob}\left\{A / w_{j}\right\}$, dado que o estado de vento considerado é único (probabilidade do único estado ' $j$ ' igual a 100\%), seguido do cálculo de GRA conforme (5). A função objetivo é modelada como:

$$
\text { Min } F O B=P E N /\left(\sum_{i=1}^{N} G R_{i}+G R A\right)
$$

No denominador de (6), tem-se a taxa de disponibilidade de geração, que deve ser maximizada. Portanto, o objetivo é minimizar a $F O B(6)$. Destaca-se que os índices $G R_{i}$ são minimizados para todas as conexões ' $i$ ', ou seja, considerando-se todas as falhas simples possíveis nestas conexões. A presença de Pen no numerador da função objetivo visa garantir as restrições apresentadas na seção 2.1 através da minimização da penalidade aplicada no caso de violação de alguma restrição.

\section{METODOLOGIA PROPOSTA}

A metodologia de otimização proposta para otimização de roteamento de parques eólicos offshore, sob os critérios de custo e confiabilidade, baseia-se na aplicação do algoritmo metaheurístico denominado ecolocalização de morcegos, ou do inglês Bat Algorithm (BA) (Yang et al., 2010). Este visa emular o comportamento de localização de morcegos e considera dois parâmetros a serem calibrados pelo usuário: a amplitude da onda sonora e a taxa de emissão da mesma. Tais parâmetros se iniciam com valores altos e baixos, respectivamente, até encontrar a presa (objetivo), quando então as taxas começam a reduzir e aumentar, respectivamente, determinando as fases de busca global e local. O pseudocódigo do algoritmo BA está apresentado na Fig. 2, segundo (Yang et al., 2010).

O algoritmo BA inicia-se determinando os parâmetros: número de morcegos $(\eta)$, taxa de redução da amplitude do pulso sonoro $(\alpha)$ e taxa de aumento da emissão do mesmo $(\lambda)$. Inicializam-se os morcegos e então avalia-se os mesmos segundo a função de mérito ou objetivo, buscando encontrar o indivíduo detentor da melhor solução. O processo iterativo do algoritmo pode ter como critério de parada o número máximo de iterações, estagnação máxima ou ambos, como é o caso do presente trabalho. A atualização de velocidade é dada segundo a linha 7 do pseudocódigo, onde ' $h$ ' representa a iteração em questão, ' $i$ ' é o índice do morcego avaliado, $x_{*}^{\text {h }}$ 
representa o melhor morcego da iteração corrente e $\beta$ é um valor aleatório entre 0 e 1.

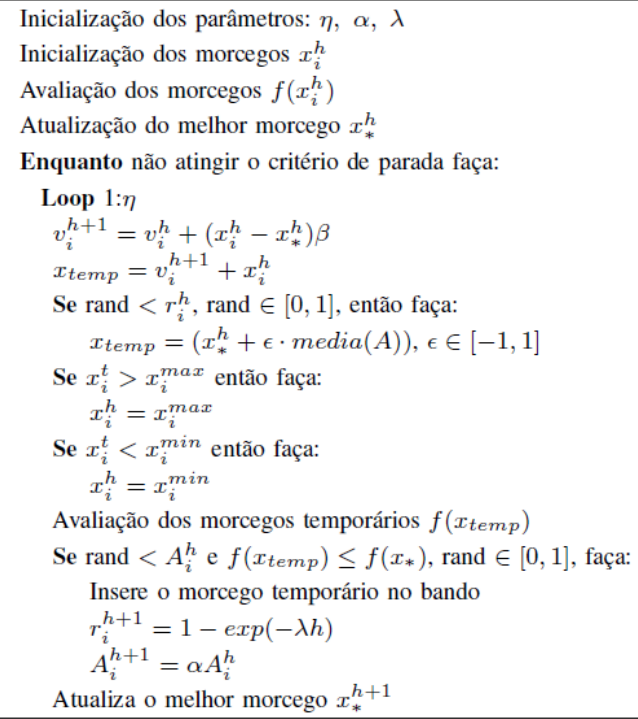

Fig. 2: Pseudocódigo do algoritmo BAT

É definido um morcego temporário sendo a posição do morcego avaliado acrescida do vetor velocidade. Gera-se um número aleatório que irá definir o comportamento da busca local. Caso o número seja menor que a taxa de emissão do morcego da iteração corrente, a busca local assume um comportamento em que o morcego temporário é substituído por um morcego criado sendo uma pequena distorção da posição do melhor morcego até o momento, conforme a linha 8 do pseudocódigo. Na equação, $\epsilon$ representa um número no intervalo $[-1,1]$ e $A_{m}$ é a amplitude média dos pulsos de onda sonora.

Caso os morcegos estejam fora das faixas aceitas para as variáveis da otimização, esses indivíduos são trazidos para o limite inferior ou superior das variáveis de otimização, avaliados conforme o critério de mérito ou função objetivo considerada.

Na busca global, um segundo número aleatório é gerado e comparado com a amplitude do pulso de onda. Caso esse número seja inferior à amplitude, o morcego temporário é incluído ao conjunto da população de indivíduos. As taxas de emissão de pulso e a amplitude de pulso são atualizadas conforme as linhas 18 e 19 do pseudocódigo, respectivamente.

Algumas modificações no algoritmo BA são propostas neste trabalho em relação ao algoritmo proposto inicialmente por (Yang et al., 2010). A primeira modificação é a condição para ocorrer a busca global. Neste trabalho, além do número aleatório ser maior que a amplitude do pulso $(r)$, é proposta a condição de ocorrer a busca global também caso o morcego temporário obtenha uma solução melhor que a solução do melhor morcego até a iteração corrente. A segunda modificação consiste em fazer com que a taxa de emissão de pulso decaia com o aumento do número de estagnações do morcego avaliado, e não com a evolução das iterações conforme algoritmo original. Estagnações são o número de iterações em que o morcego em questão não se tornou o melhor indivíduo da população. Com a população formada, avalia-se as soluções para identificar se o valor da melhor solução deve ser atualizado.

Outra modificação proposta no trabalho é arredondar os valores do vetor de velocidades obtidos para os morcegos antes de serem avaliados, uma vez que o problema tratado no presente trabalho é de otimização discreta (conexão ou não entre dois WT's). A atualização de velocidades é feita conforme (7).

$$
v_{i}^{h+1}=\operatorname{round}\left(v_{i}\right)+\operatorname{round}\left[\left(x_{*}^{h}-x_{i}^{h}\right) \cdot \beta\right]
$$

A busca local, por sua vez, foi modificada de forma a ocorrer o seguinte: i) caso a estagnação do melhor morcego seja menor do que $80 \%$ da estagnação máxima, um sorteio é feito para determinar quantas variáveis serão alteradas e outro para obter o novo valor das mesmas; ii) caso a estagnação esteja entre $80 \%$ e $95 \%$ da máxima, um sorteio determina qual variável sofre modificação e outro se será adicionada ou retirada uma conexão da variável em questão; iii) caso a estagnação seja superior a $95 \%$ da máxima, verifica-se na topologia problemas de conexão que são modificados de forma aleatória. Um exemplo do 'Caso (iii)' é apresentado na Fig. 3, em que uma possível solução para evitar o ilhamento seria conectar os WT's '2' e '4'. Entretanto, buscando a minimização do comprimento, a conexão mais adequada seria entre 2 e 3. Caso o problema seja identificado, um sorteio é realizado para corrigi-lo.

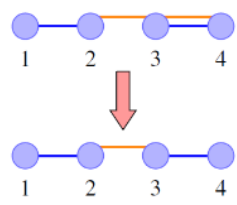

Fig. 3: Exemplo de possível problema obtido e sua correção

$\mathrm{Na}$ aplicação em questão no presente trabalho, um morcego artificial é codificado através de um vetor inteiro de tamanho $N=30$, onde o $i$-ésimo valor do vetor representa a $i$-ésima conexão entre dois geradores. Portanto, trata-se de um vetor com 30 variáveis inteiras. Destaca-se que a metodologia proposta gera apenas topologias candidatas radiais, visto ser uma topologia característica nesse tipo de sistema e visando minimizar os custos com cabeamento.

\section{ESTUDOS DE CASOS}

\subsection{Minimização do Comprimento de Cabos Utilizando BA}

Nesta etapa, o objetivo foi aplicar o algoritmo BA, conforme explicitado na seção 3, na minimização do comprimento dos cabos utilizados. A cada solução de topologia de roteamento entregue pelo algoritmo otimizador, a função objetivo aplicada foi a mencionada na seção 2.1. Portanto o objetivo é minimizar o comprimento dos cabos respeitando as topologias possíveis e viáveis radiais no parque eólico considerado. Foram utilizadas populações de tamanhos 100, 200, 500 e 1000 com diferentes valores de $\alpha$ : 0,85; 0,9 e 0,95 . 
A variável $\lambda$ assumiu valores de 0,$05 ; 0,1$ e 0,15 . A Fig. 4 apresenta a eficiência obtida para cada combinação de parâmetros realizada.

\begin{tabular}{|c|c|c|c|c|}
\hline \multirow{2}{*}{$\boldsymbol{I}$} & $\boldsymbol{\lambda}$ & \multicolumn{3}{|c|}{$\boldsymbol{\alpha}$} \\
\cline { 3 - 5 } & & 0,85 & 0,9 & 0,95 \\
\hline \multirow{3}{*}{100} & 0,05 & 85 & 80 & 77 \\
\cline { 2 - 5 } & 0,1 & 82 & 79 & 88 \\
\cline { 2 - 5 } & 0,15 & 82 & 93 & 89 \\
\hline \multirow{3}{*}{200} & 0,05 & 80 & 81 & 88 \\
\cline { 2 - 5 } & 0,1 & 87 & 81 & 82 \\
\cline { 2 - 5 } & 0,15 & 88 & 83 & 82 \\
\hline \multirow{3}{*}{500} & 0,05 & 80 & 81 & 79 \\
\cline { 2 - 5 } & 0,1 & 85 & 89 & 79 \\
\cline { 2 - 5 } & 0,15 & 78 & 76 & 81 \\
\hline \multirow{3}{*}{1000} & 0,05 & 86 & 86 & 86 \\
\cline { 2 - 5 } & 0,1 & 81 & 84 & 85 \\
\cline { 2 - 5 } & 0,15 & 80 & 81 & 77 \\
\hline
\end{tabular}

Fig. 4: Eficiência percentual obtida.

O problema possui um total de $30^{31}$ (aproximadamente 5,5 x $10^{44}$ ) combinações possíveis de soluções. O valor obtido para a função objetivo foi um comprimento total de 7.400 metros. A topologia obtida está representada na Fig. 5. O ' $x$ ' representado com número 1 localiza a posição da subestação. É necessário salientar que existem várias topologias que satisfazem o mínimo comprimento. A ligação entre os WT's 4 e 14 poderia ser substituída por $2-12,3-13,5-15,6-16,7$ $17,8-18,9-19,10-20$ ou 11-21 que obteria o mesmo valor ótimo encontrado. A mesma observação vale para a ligação 17-27 em relação às ligações 12-22, 13-23, 14-24, 15-25, 1626, 18-28, 19-29, 20-30 e 21-31.

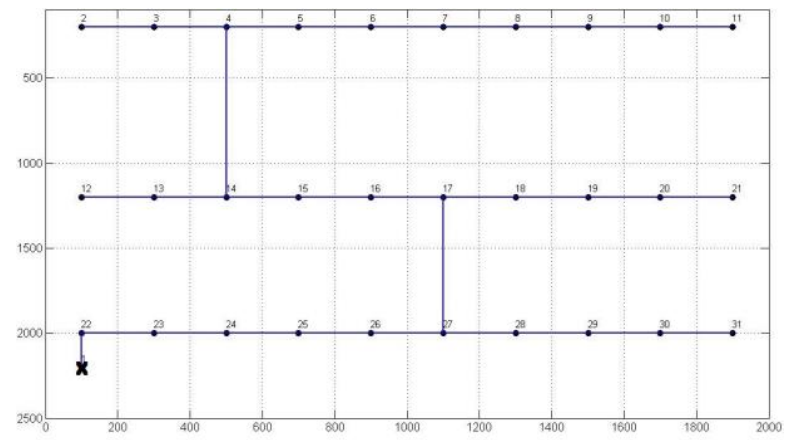

Fig. 5: Topologia obtida minimizando comprimento de cabos

Pode-se notar que minimização de comprimentos de cabo não é um critério suficiente para projeto da topologia do roteamento, uma vez que outros critérios como confiabilidade são de extrema relevância para o custo de implantação e operação do sistema eólico. Além da otimização, outra alternativa, para encontrar o menor custo em cabeamentos, seria utilizando um algoritmo de caminho mínimo. Entretanto, utilizou o BAT como uma avaliação do algoritmo e sua capacidade de obter a melhor solução. O foco é apresentar e analisar os resultados de uma abordagem em otimização uniobjetiva.

\subsection{Maximização da Confiabilidade Utilizando BA}

O algoritmo BA é aplicado e, a cada solução encontrada, a avaliação é dada pela função objetivo modelada na seção 2.2. O objetivo é encontrar uma topologia que seja viável e que maximize o índice de confiabilidade proposto na seção mencionada, simulando-se falhas nos cabos com contingência 'N-1' (uma falha para cada estado avaliado). A simulação foi executada 10 vezes considerando os parâmetros fixos, devido ao tempo computacional, iguais a: $\alpha=0,9 ; \lambda=0,1$ e $\eta=100$ indivíduos. A eficiência obtida foi de $80 \%$ das simulações executadas e o resultado para topologia encontrada está representado na Fig. 6.

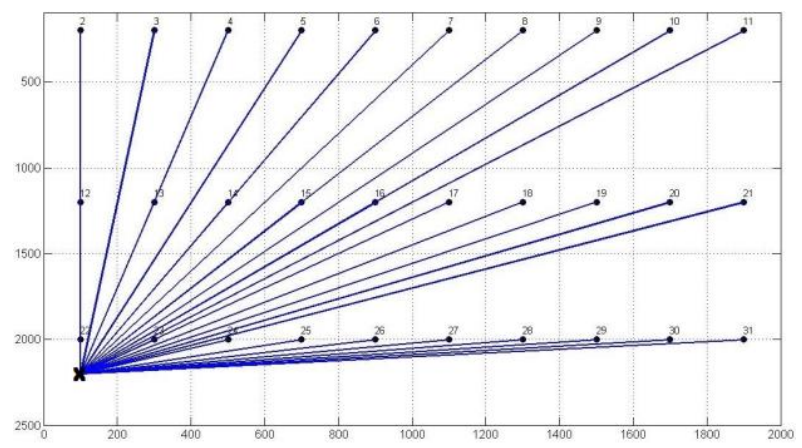

Fig. 6: Roteamento via maximização do índice GR

$\mathrm{O}$ valor ótimo encontrado foi de GRA $=1$ pois em todos os cenários todos os índices GR obtidos foram superiores ao GR crítico. O índice GR foi de 0,9638 para as 10 primeiras falhas (referentes aos cabos dos WT's 2 a 11), para as próximas 10 falhas nos cabos referentes as turbinas de 12 a 21 o índice foi de $\mathrm{GR}=0,9673$ e as últimas falhas referentes aos cabos dos WT's 22 a 31, GR foi igual a 0,9689. Essa diferença obtida para os índices GR se dá pelas diferentes potências extraídas pelos WT's, uma vez que o vento se dá na direção norte-sul, o wake effect influencia na potência captada pelos WT's à jusante de outros. A potência extraída considerando o efeito de enfraquecimento para os WT's de 2 a 11, 12 a 21 e 22 a 31 foram de, respectivamente, 5,$184 ; 4,672$ e 4,454 kW. O somatório dos índices GR obtidos foi de 29 e a média dos índices foi 0,9667 . Assim, o valor da função objetivo foi de 0,0333 .

A topologia ótima encontrada vai ao encontro do método proposto. Ao conectar todos os WT's à subestação, cada falha de cabos obtida só vai interferir no seu WT em questão, mantendo o restante do parque eólico em funcionamento. Ao levar em consideração apenas a confiabilidade, a topologia encontrada viabiliza a máxima confiabilidade do parque na análise de falhas de barramento, mas inviabiliza custos ligados ao tamanho dos cabos, por exemplo, o comprimento total de cabos obtidos foi de 46,201 km. A otimização uniobjetiva não contempla todos os aspectos que devem ser otimizados e levados em consideração em um projeto de um sistema eólico offshore.

\subsection{Minimização de Comprimento de Cabos e Maximização da Confiabilidade Simultaneamente Utilizando BA}

Nesta etapa, a avaliação é dada por uma função objetivo híbrida no qual o objetivo é maximizar a confiabilidade da geração e minimizar a distância dos cabos condutores. Ou seja, deseja-se o menor custo com cabos condutores obtendo a maior confiabilidade possível medida a partir do índice GR. 
Dessa forma, a terceira modelagem é uma forma híbrida das duas funções objetivo (3) e (6).

$$
\operatorname{Min} F O B=P E N \cdot L /\left(\sum_{i=1}^{N} G R_{\mathrm{i}}+G R A\right)
$$

Colocando-se o comprimento no numerador e o cálculo da confiabilidade no denominador, ao buscar-se minimizar a função objetivo, automaticamente busca-se a maior confiabilidade proposta bem como o menor custo de cabeamento. O objetivo é encontrar uma topologia que seja viável e que maximize o índice de confiabilidade bem como minimize o comprimento dos condutores utilizados de forma simultânea.

A simulação foi feita com o tamanho das populações assumindo os valores de 100; 200 e 500. O parâmetro $\alpha$ assumiu valores de 0,$85 ; 0,9$ e 0,95 . O parâmetro $\lambda$, por sua vez, assumiu os valores de 0,05 ; 0,1 e 0,15 . Para cada uma das combinações entre número de indivíduos, $\alpha$ e $\lambda$, a simulação foi executada 10 vezes. Assim, no total, a simulação executou 270 vezes alternando os parâmetros mencionados. A melhor topologia encontrada está exposta na Fig. 7. O valor da função objetivo foi de 270,2702 e o comprimento dos cabos obtidos foi de 7527,2677 metros.

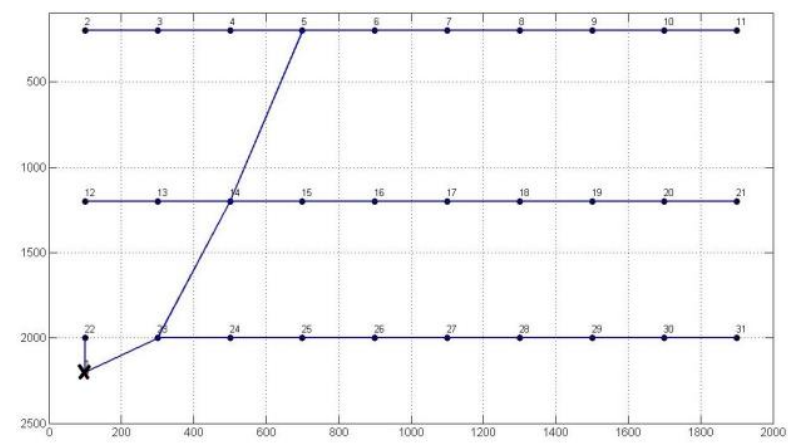

Fig. 7: Roteamento via maximização do índice GR e minimização da distância

A topologia encontrada condiz com o esperado já que apresenta uma forma híbrida se comparada com a mesma obtida maximizando confiabilidade ou minimizando comprimento, individualmente. Apresenta os valores de confiabilidade e de distância de condutores entre os limites encontrados na otimização individualizada, coerente com o esperado.

\section{CONCLUSÕES}

Este trabalho apresentou a otimização do roteamento interno de cabos de um parque eólico offshore através dos critérios da minimização de custos de cabeamento e maximização de confiabilidade. Pode-se concluir que os resultados foram coerentes com o esperado, uma vez que as topologias encontradas satisfizeram o objetivo buscado sob cada critério. Pode-se inferir que otimizar comprimento ou confiabilidade não são critérios que possam ser levados individualmente em consideração para o projeto desse tipo. A otimização deve ter abordagem multiobjetiva, também simulada neste trabalho. Os diferentes critérios conduzem a configurações de topologia bastante diversas entre si, demonstrando que ao analisar um requisito individualmente, o resultado obtido é específico para o critério utilizado e não representa uma solução holística incluindo todos os critérios, indicando a necessidade de utilização de modelos multiobjetivos.

\section{AGRADECIMENTOS}

Este trabalho foi desenvolvido dentro do projeto "Estudo de Metodologias para Conexão de Usinas Eólicas Offshore de Grande e Pequeno Porte e Aspectos Elétricos e Energéticos" PD-0553- 0045/2016 no âmbito do programa de P\&D do sistema elétrico regulado pela ANEEL. Os autores desse artigo agradecem o apoio da PETROBRAS, FAPEMIG, CAPES, CNPq, INERGE, PPEE-UFJF e do GOHB - UFJF.

\section{REFERÊNCIAS}

Associação Brasileira de Energia Eólica, abeeolica.org.br, 20 de Abril de 2019

Huang L., Fu Y., Guo X. (2009) "Optimization of Electrical Connection Scheme for Large Offshore Wind Farm with Genetic Algorithm" International Conference on Sustainable Power Generation and Supply, 2009

Li, Dong D., Chao H., Yang Fu. (2008) "Optimization of internal electric connection system of large offshore wind farm with hybrid genetic and immune algorithm." Electric Utility Deregulation and Restructuring and Power Technologies, 2008.

Mahidhar N., Shirshak K. D. (2008) "Optimal Design of an Offshore Wind Farm Layout" International Symposium on Power Electronics, 2008 IEEE.

Menghua Z., Zhe C., Blaabjerg F. (2007) "Generation Ratio Availability Assessment of Electrical Systems for Offshore Wind Farms" IEEE Transactions On Energy Conversion, Vol. 22, N0 3. (2007)

Pookpunt S., Ongsakul W. (2013) “Optimal placement of wind turbines within wind farm using binary particle swarm optimization with time varying acceleration coefficients", Renewable Energy, 55, 266-276

Smail H., Alkama R., Medjdoub A. (2018) "Optimal design of the electric connection of a wind farm" Energy 165 (2018) 972-983

World Wind Energy Association (WWEA). wwindea.org, 20 de Abril de 2019

Wu, Y. K., Lee, C. Y., Chen, C. R., Hsu, K. W., ans Tseng, H. T. (2014). "Optimization of the wind turbine layout and transmission system planning for a large-scale offshore windfarm by AI technology". IEEE Transactions on Industry Applications, 50(3), 2014.

Xiaoqing H., Jing G., Peng W. Yanbing J. (2011) “Adequacy Study of Wind Farms Considering Reliability and Wake Effect of WTGs" Power and Energy Society General Meeting, 2011 IEEE (pp 1-7)

Yang, H., Xie, K., Tai, H. M., e Chai, Y. (2016). "Wind farm layout optimization and its application to power system reliability analysis". IEEE Transactions on Power Systems, 31(3), 2016

Yang, Xin-She. (2010) "A new metaheuristic bat-inspired algorithm." Nature inspired cooperative strategies for optimization (NICSO 2010). 65-74. 